\title{
Pulse Oximetry Screening in newborn for early detection of Critical Congenital Heart Disease - A review
}

\author{
SUKHENDU SHEKHAR SEN ${ }^{1}$, TASKINA MOSLEH ${ }^{2}$, ISMAT JAHAN ${ }^{3}$, SANJOY KUMER DEY ${ }^{4}$
}

\begin{abstract}
Approximately 9 of 1000 live births have congenital heart disease, of these $\sim 25 \%$ have critical congenital heart disease (CCHD). Delayed or missed diagnosis of CCHD may result in acute cardiovascular collapse. Echocardiography is a necessary diagnostic tool but it's cost is a severe limitation as a universal screening tool. Clinically undetectable hypoxemia is present to some degree in most CCHD and this technique can be used as a screening method for early detection. In 2012, a systematic review involving over 300,000 babies reported that pulse oximetry test is highly specific (99.9\%) and moderately sensitive (76.5\%) with a low false positive rate $(0.05 \%)$. The test is simple, feasible, cost effective, highly specific and sufficiently sensitive to qualify for screening test. Parents and caregivers should be informed that pulse oximetry cannot detect all cases of CCHD. A positive test result needs to be confirmed by echocardiography, which is considered as the definitive diagnostic modality. Whenever possible, the echocardiogram should be interpreted by pediatric cardiologists.
\end{abstract}

Key words: Pulse oximetry, Critical Congenital Heart disease

\section{Introduction}

Congenital heart disease (CHD) is the most common cause of infant death associated with congenital malformation in developed world. ${ }^{1}$ Approximately 9 of 1000 live births have congenital heart disease. ${ }^{2}$ Of these $\sim 25 \%$ have critical congenital heart disease (CCHD), defined as a condition requiring surgical or catheter intervention in the first year of life. ${ }^{3}$ Early detection of $\mathrm{CCHD}$ might improve the outcome through timely appropriate treatment ${ }^{4}$ while delayed or missed diagnosis may result in acute cardiovascular collapse, acidosis and death corresponding to changes in pulmonary vascular resistance and closure of ductus arteriosus. ${ }^{5}$ Unrecognized CCHD in turns leads to a huge medical burden, economic burden and mental burden for the family and for society. ${ }^{4}$ It accounts for $1-2$ deaths per 100,000 livebirths. ${ }^{5}$

1. Assistant Professor, Department of Paediatrics, Chattagram Maa-O-Shishu Hospital Medical College, Agrabad, Chittagong.

2. Medical officer, Department of Neonatology, Bangabandhu Sheikh Mujib Medical University (BSMMU), Dhaka, Bangladesh.

3. Medical officer, Department of Neonatology, Bangabandhu Sheikh Mujib Medical University (BSMMU), Dhaka, Bangladesh.

4. Associate Professor, Department of Neonatology, Bangabandhu Sheikh Mujib Medical University (BSMMU), Dhaka, Bangladesh.

Correspondence: Dr. Sukhendu Shekhar Sen, E-mail: sukhendusen@gmail.com.
Bangladesh is well ahead in the management of congenital heart defects despite scarcity of precise disease burden. Two hospital based study findings are suggestive of variable prevalence of congenital heart disease (7.8 and 25/1000 live births respectively) among Bangladeshi population which mandated to carry out heart defects related documentation. ${ }^{6,7}$

Contemporary routine screening for congenital heart disease depends on a mid-trimester anomaly scan, involving imaging of the heart chambers, and a postnatal cardiovascular system examination. ${ }^{8}$ The sensitivity of $\mathrm{CHD}$ detection by anomaly scan is highly variable, depending on operator expertise, gestational age, fetal position, and the type of cardiac defect. ${ }^{9}$ Even when foetal ultrasound is routinely performed, fewer than $50 \%$ of cases of CCHD are identified. Detection rate by postnatal clinical examination alone is also low leaving up to one-third of babies undiagnosed at the time of discharge with critical heart defects. ${ }^{8}$ Echocardio-graphy is an obligatory diagnostic tool but the cost is a severe limitation as a universal screening tool. Moreover, echocardiography has a high frequency of false positive results usually related to the transitional circulation and there may be inadequately trained personnel to perform the test with reasonable accuracy. ${ }^{10}$ 
Neonates with CCHD can be diagnosed on the basis of physical findings. These findings are not always evident before hospital discharge. A recent study from the United Kingdom suggested that $25 \%$ of infants with CCHD were not diagnosed with heart disease until after discharge from the newborn nursery. 11 Newborns with CCHD are susceptible to profound, sudden worsening in clinical status in the first days and weeks of life. ${ }^{12}$ The newborn hospitalization provides a potential scope for caregivers to identify CCHD lesions hence to avoid hemodynamic embarrassment. ${ }^{12}$

Pulse oximetry screening for early detection of CCHD was first reported more than a decade back with the principle that some degree of clinically undetectable hypoxemia is present in most of the CCHD. Children with mild hypoxemia, with arterial oxygen saturation of $80 \%-95 \%$ may not have visible cyanosis. Moreover identification of cyanosis is particularly problematic in dark skinned baby. Pulse oximetry is considered a vital sign equivalent in importance to pulse, respirations and blood pressure. Contemporary use of pulse oximetry contributes to heightened recognition of congenital heart disease in neonate. ${ }^{13}$

In current era, congenital heart surgery allows for repair or palliation of nearly all types of congenital heart malformation. ${ }^{14,15}$ Intervention is typically performed in the first weeks of life to optimize hemodynamic and prevent end-organ injury associated with delayed diagnosis. ${ }^{12}$ There is a wide gap between the developed and developing countries regarding pediatric cardiac care. Without early recognition, diagnosis and treatment, a majority of infants and children with congenital heart disease die in their first month of life in developing countries. ${ }^{16}$ This review will document the most recent data regarding pulse oximetry as universal screening for early detection of CCHD.

\section{Recent evidences}

Challenges were remaining regarding potential role of pulse oximetry screening for detection of CCHD until past few years. According to a scientific statement from the American Heart Association (AHA) and American Academy of Pediatrics more researches were encouraged to carry out to establish pulse oximetry screening performance involving larger populations and across a broad range of newborn delivery systems. ${ }^{12}$ Recently, several large European studies ${ }^{13,14,16-18}$ have strengthened the case and demonstrated that pulse oximetry as an adjunct to existing screening can increase $\mathrm{CCHD}$ detection rates to well over $90 \%$. ${ }^{19}$ Importantly, most studies also report detection of non-critical congenital heart defects
(CHD) or serious non-cardiac illness such as congenital pneumonia, early-onset sepsis and pulmonary hypertension. As some of these conditions are potentially as lethal as a CCHD if undiagnosed (eg, group B streptococcal pneumonia), this is a key additional advantage of the pulse oximetry. ${ }^{19}$

In 2009, a Swedish prospective study published a test accuracy involving about 40,000 babies including an assessment of both preductal and postductal saturation. They reported the sensitivity of $62 \%$ and specificity of $99.8 \%$. Screening was performed at a median age of 38 hours with a low false positive rate of $0.17 \%$. They also found pulse oximetry as an effective adjunct to clinical examination and the combined detection rate of $\mathrm{CCHD}$ was $92 \%$. $^{18}$

In the subsequent year, a German study by Riede et al ${ }^{19}$ screened over 40,000 babies using only postductal saturations between $24 \mathrm{hrs}$ and $72 \mathrm{hrs}$ of age. They reported the sensitivity of $78 \%$ and specificity of $99.9 \%$ and a false positive rate of $0.1 \%$.

Pulse oximetry study from the United Kingdom in the year 2011 has enrolled more than 20,000 babies to establish accuracy, acceptability and cost effectiveness of pulse oximetry screening for detection of CCHD. Reported sensitivity for detecting CCHD was $75 \%$, specificity $99.1 \%$ and false positive rate $0.8 \%{ }^{3}$

A recent systematic review and meta-analysis of previous good quality 13 studies in 2012 involving a large cohort of 2,30,000 babies have reinforced the existing evidence regarding accuracy of the pulse oximetry as an effective adjunct to existing screening. They concluded that it is highly specific (specificity $99.9 \%, 95 \% \mathrm{Cl} 99.7$ to 99.9 ), moderately sensitive (sensitivity $76.5 \%, 95 \% \mathrm{Cl} 67.7$ to 83.5 ) test with a low false positive rate $(0.14 \%)$ which can be reduced more $(0.05 \%)$ if the test is done after $24 \mathrm{hrs}$ of age. All these findings suggested that pulse oximetry met the criteria for universal screening. They also concluded that, in view of number of babies studied over series of researches, it is unlikely that any further work would demonstrate substantially different results. ${ }^{1}$

\section{Screening approach}

Currently, children with CCHD are diagnosed usually on physical examination findings (e.g. heart murmur, tachypnoea, cyanosis). Chest X-ray, ECG, Color Doppler echocardiogram, Arterial Blood Gas analysis, pulse oximetry are only done if the baby is symptomatic or has increased risk of CCHD (positive family history, chromosomal anomaly etc). In USA, delayed or missed diagnosis of CCHD may occur in 7/1000 live births results in sudden and profound worsening of 
clinical status, and increase in morbidity and mortality. ${ }^{12}$

As one of the most important non-invasive advances of clinical examination in recent year's pulse oximetry screening is being adopted by individual hospitals and as national policy. It has been recommended as uniform screening panel in year 2011 by US Health and Human services secretary. Most infants in Switzerland, Abu Dhabi and Ireland are also routinely screened. ${ }^{20-23}$

Some degree of heterogeneity exists between the screening pathways in published studies, particularly in timing of the screening, postductal or preductal and postductal saturation measurement ; saturation threshold for a positive test. ${ }^{21}$

Recent studies showed that later screening (> $24 \mathrm{~h}$ ) has a lower false positive results than early screening $(<24 \mathrm{~h})^{1,12}$ Although later testing may miss the opportunity for intervention before spontaneous closing of the ductus arteriosus ${ }^{4}$. Approximately half of the babies with $\mathrm{CCHD}$ screened after $24 \mathrm{hr}$ present with symptoms prior to screening. And up to $10 \%$ of them may present with collapse in hospital. ${ }^{24}$ Taking circulation collapse and clinical deterioration in consideration ,pulse oximetry screening test should be completed between 24 and 48 hrs of age or shortly before discharge if $<24 \mathrm{hrs}$ of age. ${ }^{4}$

Pulse oximetry screening is recommended to be conducted with motion-tolerant pulse oximeters ${ }^{4}$.that report functional oxygen saturation. No standards have been developed yet regarding motion tolerance. Anew guidance document on the safety and effectiveness of pulse oximeters is being developed by FDA. Having specific FDA-cleared labeling and conformance to the relevant standard will be an important strategy for ensuring that appropriate devices are used for screening. ${ }^{25}$

Screening should be done in a quite room, while the infant is awake and calm because low oxygen saturation caused by hypoventilation during sleep may lead to false positive results ${ }^{4}$. Infant's right hand and both feet should be clean and dry, as dried blood can affect the pulse oximetry reading. Probe should be placed on the outside fleshy part of the infant's hand and foot. Probes with close coupling to skin (ie, taped rather than clamped) provide better performance for oximetry monitoring in newborns. Furthermore, to optimize valid screening, manufa-cturer recommended pulse-oximeter-probe combinations should be used. ${ }^{25}$

Position of pulse oximetry probe with respect to the right hand and either foot is another point of variation among published studies which reflects preductal and postductal measurement of oxygen saturation respectively. ${ }^{4,12}$ In some situations as transposition of great arteries with right to left shunting at the level of ductus arteriosus, cyanosis present in the upper part of body. So, its more accurate to measure it both preductally and postductally; although most studies measured saturation on either foot. ${ }^{4}$ Two recent studies showed that if only post-ductal measurement is used instead of both pre-ductal and post-ductal measurements, there is risk of missing babies with $\mathrm{CCHD}{ }^{3,18}$ It is possible that the inclusion of both upper and lower $\mathrm{Spo}_{2}$ measurements would result in a significant higher false positive rate. Moreover it would be more time consuming to screen a single neonate. Therefore, a single lower extremity reading would appear to be most appropriate for the purpose of largescale screening. ${ }^{12}$

It is important to establish a cutoff threshold for an abnormal $\mathrm{Spo}_{2}$. Other factors being constant a high threshold will increase sensitivity and at the same time decrease specificity. Whereas, setting the $\mathrm{Spo}_{2}$ cutoff value closer to the normal value will decrease the number of false negative screening results at the cost of increasing the number of false positive screening results. ${ }^{12}$ The limit for a positive test varies between $92-95 \%$ with the majority of studies using less than $95 \% .{ }^{21}$

On the basis of new data from the large population based screening activities in Sweden and England, the work group developed a recommendation for screening that was based on what was shown to be effective in those studies. A screen result would be considered positive if -1) Any oxygen saturation measure is $<90 \%, 2$ ) oxygen saturation is $<95 \%$ in both extremities on three measures, each separated by 1 hour, or 3 ) There is a $>3 \%$ absolute difference in oxygen saturation between the right hand and foot on 3 measures, each separated by 1 hour. Any screening that is $\geq 95 \%$ in either extremity with $\leq 3 \%$ absolute difference in oxygen saturation between upper and lower extremity would be considered a "pass" result and screening would end. ${ }^{25}$ Figure -1 . Additionally, the threshold for a positive pulse-oximetry screening result should be lower in the plateau environments at different altitude areas. But the normal values of $\mathrm{Spo}_{2}$ at different altitudes have not been established. If the pulse oximetry screening for CCHD would be carried out in the plateau areas, the normal values should be established first. ${ }^{4}$

\section{Cost-effectiveness}

Implementation of universal pulse oxymetry screening is feasible and achievable because screening can be 


\section{Algorithm for Pulse Oximetry Screening for CCHD: ${ }^{25}$}

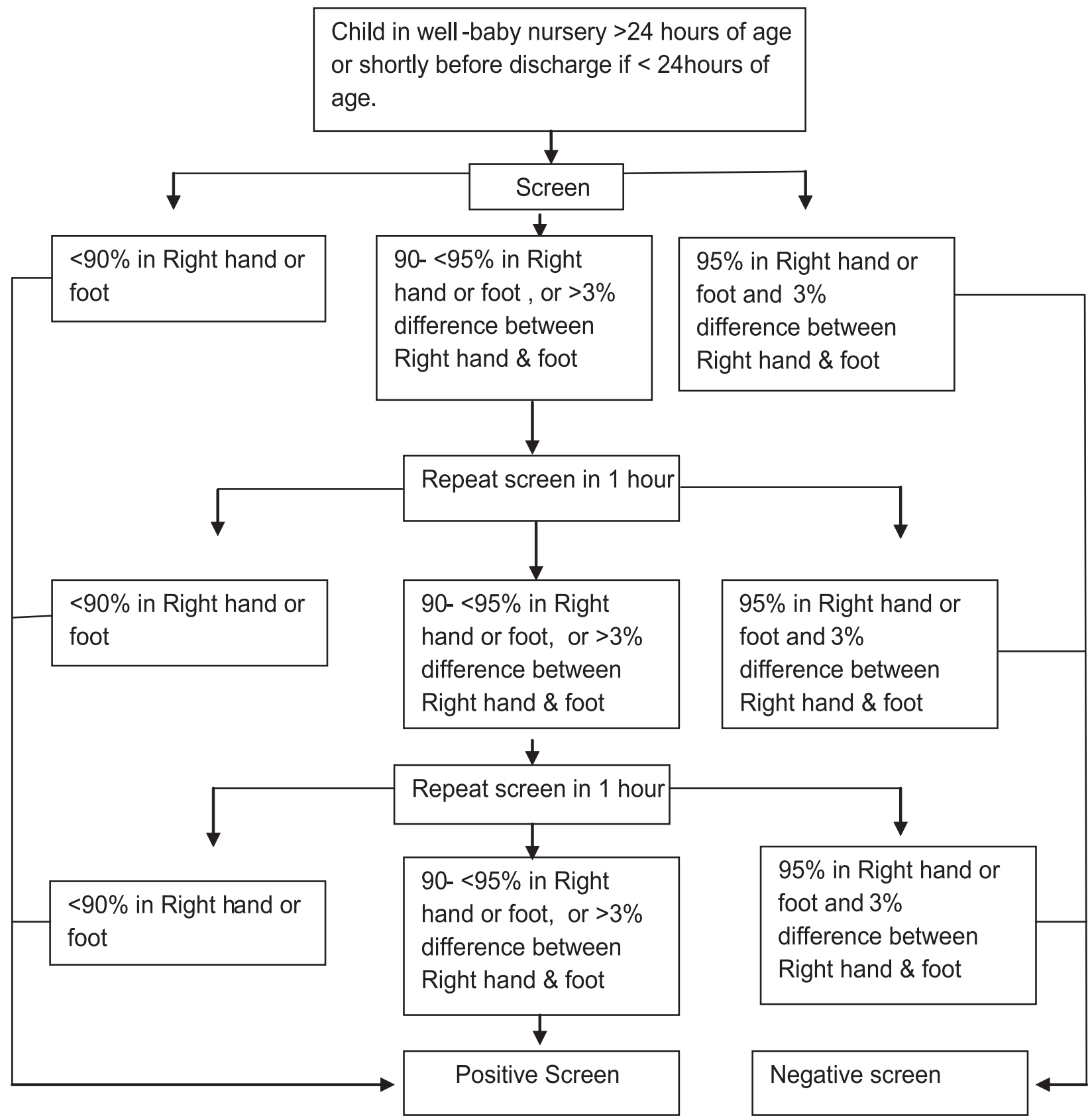

Fig.-1: Pulse oximetry monitoring protocol based on results from the right hand and either foot.

achieved without additional staff and without over burdening clinical services. Care of a case of circulatory collapse resulting from an undiagnosed CCHD may exceed the cost of screening of two thousand newborns. ${ }^{26}$ Addition of pulse oximetry screening with clinical examination is cost effective with no risk of harm, and diagnostic echocardio-graphy should be performed in all test positive cases. In situation with a lower false positive rate and a lower antenatal detection, the cost effectiveness is likely to improved. ${ }^{27}$ In Minnesota, USA, implementation of Critical Congenital Heart disease screening reported mean nursing time for screening was 5.5 minute (range 1 40 minutes), and cost of reusable probe was $\$ 0.30$ / 
screen (probe price is $\$ 300.00$, that can be used for up to 1000 screens) and cost of straps was $\$ 0.12$ I patient ( $\$ 0.06$ per strap * 2 ). The cost of each neonatal echocardiogram was $\$ 1300$ at that period of time. ${ }^{28}$

\section{Limitations}

The sensitivity of pulse oxymetry test is around $75 \%$, it means that about a quarter of babies with $\mathrm{CCHD}$ may not be detected by using this screening test. The commonest lesions missed are those causing obstruction to aortic flow (e.g. coarctation of aorta and interrupted arch), which are not necessarily be associated with hypoxemia. Surprisingly, this screening test may miss transposition of great arteries occasionally, which is usually presented with cyanosis. ${ }^{3,18,26}$ Both the parents and clinical staffs should be aware of the limitations of this screening test. Parents and caregivers should be informed that pulse oximetry cannot detect all cases of $\mathrm{CCHD}$, and hence a negative test result does not exclude the possibility of heart disease.

\section{Conclusion}

The pulse oximetry test is simple, feasible, cost effective, highly specific and sufficiently sensitive to qualify for screening test. It is also acceptable to parents and clinical staffs. A recent Lancet editorial described the technique as "a new milestone in the history of congenital heart disease". ${ }^{29}$ A positive test result need to be confirmed by echocardiography, which is considered as the definitive diagnostic modality. Whenever possible, the echocardiogram should be interpreted by pediatric cardiologists as accuracy of pediatric echocardiograms by adult cardiologists is low. ${ }^{30}$

Pulse oximetry improves detection rates of critical CHDs. The majority of critical cases missed by pulse oximetry (and by other screening methods) are associated with obstruction of the aortic arch as these conditions are often not associated with hypoxemia. Further investigation of other oximetry techniques, such as perfusion index, may enhance the detection rates for these lesions. Further research should be conducted with mothers of different ethnicities to gain a greater understanding of factors limiting participation and satisfaction with testing. Research should also be conducted with mothers given false positive results at the time of discharge from hospital to verify whether they experience heightened anxiety at this point.

\section{References}

1. Thangaratinam S, Brown K, Zamora J, Khan KS, Ewer AK. Pulse oximetry screening for critical congenital heart defects in asymptomatic newborn babies: a systematic review and metaanalysis. Lancet. 2012;379:2459-64.

2. Botto LD, Correa A, Erickson JD. Racial and temporal variations in the prevalence of heart defects. Pediatrics. 2001;107.

3. Ewer AK, Middleton LJ, Furmston AT. PulseOx Study Group. Pulse oximetry screening for congenital heart defects in newborn infants (PulseOx): a test accuracy study. Lancet. 2011; 378:758-49.

4. Xiao-Jing M, Guo-Ying H. Neonatal Pulse oximetry screening improves detecting of critical congenital heart disease. Chin Med J. 2013; 126:2736-40.

5. Chang R-KR, Gurvitz M, Rodrigues S. Missed diagnosis of critical congenital heart diseas. Arch Pediatr Adolesc Med. 2008;162:969-74.

6. Islam MN, Hossain MA, Khaleque MA, Das MK, Khan MRH, Bari MS et al. Prevalence of congenital heart disease in neonate in a tertiary level hospital. NJMS. 2013;2:91-95.

7. Fatema NN, Chowdhury RB, Chowdhury L. Incidence of Congenital Heart Disease among Hospital Live Birth in a Tertiary Hospital of Bangladesh. Cardiovascular Journal. 2008;1: 14-20.

8. Hoffman JIE. It is time for routine neonatal screening by pulse oximetry. Neonatology. 2011; 99:1-9.

9. Harold JG. Screening for critical congenital heart disease in newborns. Circulation. 2014;130: e71-e81.

10. Griebsch I, Knowles RL, Brown J, Bull C, Wren C. Comparing the clinical and economic effect of clinical examination, pulse oximetry, and echocardiography in newborn screening for congenital heart defects: a probabilistic costeffectiveness model and value of information analysis. Int J Technol Assess Health Care. 2007;23:192-204. 
11. Brown KL, Ridout DA, Hoskote A, Verhulst L, Ricci M, Bull C. Delayed diagnosis of congenital heart disease worsens preoperative condition and outcome of surgery in neonates. Heart. 2006;92:1298-1302.

12. Mahle WT, Newburger JW, Matherne GP. Role of pulse oxymetry in examining newborns for congenital heart disease: a scientific statement from the AHA and AAP. Pediatrics. 2009;124: 823-36.

13. Koppel RI, Druschel C, Carter T. Effectiveness of pulse oximetry screening for congenital heart disease in asymptomatic newborns. Pediatrics. 2003;111:451-55.

14. Sharma M, Sasikumar M, Karloopa SD, Shahi BN. Prostaglandins in Congenital Heart Disease. MJAFI. 2001;57:134-38.

15. Boneva RS, Botto LD, Moore CA, Yang Q, Correa A, Erickson JD. Mortality associated with congenital heart defects in the United States: trends and racial disparities,1979-1997. Circulation. 2001;103:2376-81.

16. United Nations 2000. Millennium development goals: UN Millenium Declaration. http:// www.un.org/millenniumgoals/(accessed May14, 2015).

17. Hussain M, Tahura S, Sayeed MA, Rahman MM, Rahman MM, Kar SK. Past and present pattern of Congenital Heart Disease at Dhaka Shishu Hospital: A situation analysis. Bangladesh J Child Health. 2010;34:51-55.

18. De-Wahl Granelli A, Wennergren M, Sandberg K. Impact of pulse oximetry screening on detection of duct dependent congenital heart disease: a Swedish prospective screening study in 39821 newborns. BMJ. 2009;338:a3037.

19. Riede FT, Worner C, Dahnert I. Effectiveness of neonatal pulse oximetry screening for detection of critical congenital heart disease in daily clinical routine: results from a prospective multicenter study. Eur J Pediatr. 2010;169:975-81.

20. Turska "Kmieæ A, Borszewska" Kornacka MK, $B$ W, et al. Early screening for critical congenital heartdefects in asymptomatic newborns in Mazoviaprovince: experience of the POLKARD pulseoximetry programme 2006-2008 in Poland. Kardiologia Polska. 2012;70:370-76.

21. Ewer AK. Review of pulse oximetry screening for critical congenital heart defects in newborn infants. Curr Opin Cardiol. 2013;28:92-96.

22. Mahle WT, Martin GR, Beekman RH III, et al. Endorsement of Health and Human Services recommendation for pulse oximetry screening for critical congenital heart disease. Pediatrics. 2012;129:190-2.

23. Kuelling B, Arlettaz Mieth R, Bauersfeld U. Pulse oximetry screening for congenital heart defects in Switzerland: most but not all maternity units screen their neonates. Swiss Med Wkly. 2009; 139:699-704.

24. Ewer AK. Pulse oximetry screening for critical congenital heart defects in newborn infant: Should it be routine?. Arch Dis Child Fetal Neonatal Ed. 2013;0:F1-F3.

25. Kemper AR, Mahle WT, Martin GR, Cooley WC. Strategies for Implementing Screening for Critical Congenital Heart Disease. Pediatrics. 2011;128:1259-67.

26. Bradshaw EA, Cuzzi A, Kiernan SC. Feasibility of implementing pulse oxymetry screening in a community hospital. J Perinatol. 2012;32: 710-15.

27. Robert TE, Barton P, Auguste P. Pulse oxymetry as a screening test for congenital heart disease in newborn infants: a cost effectiveness analysis. Arch Dis Child. 2012;97:221-26.

28. Kochilas LK, Lohr JL, Bruhn E, Borman-Shoap E, Gams BL. Implementation of Critical Congenital Heart Disease screening in Minnesota. Pediatrics. 2013;132:587-94.

29. A new milestone in the history of congenital heart disease (editorial). Lancet. 2012;379:2401.

30. Ward CJ, Purdie J. Diagnostic accuracy of paediatric echocardiograms interpreted by individuals other than paediatric cardiologists. J Paediatr Child Health. 2001;37:331-46. 